08,04

\title{
Новая магнитная нецентральная примесь - кобальт в титанате стронция
}

\author{
() И.А. Случинская, А.И. Лебедев \\ Московский государственный университет им. М.В. Ломоносова, \\ Москва, Россия \\ E-mail: irinasluch@gmail.com
}

(Поступила в Редакцию 30 октября 2018 г.)

Методом XAFS-спектроскопии исследованы локальная структура и зарядовое состояние примеси кобальта в $\mathrm{SrTiO}_{3}$. Найдены условия синтеза образцов $\mathrm{SrTiO}_{3}(\mathrm{Co})$, при которых кобальт преимущественно (до 76\%) замещает атомы в узле $A$ структуры перовскита. Меняя условия синтеза, можно заметно изменять соотношение концентраций атомов кобальта, входящих в узлы $A$ и $B$. Показано, что зарядовое состояние кобальта в узле $A$ равно +2 , а в узле $B-+3$. Обнаружено, что примесь Со в узле $A$ является нецентральной примесью, а ее смещение из узла решетки составляет $1.0 \AA$. Расчеты из первых принципов показывают, что изолированный ион $\mathrm{Co}^{3+}$ в узле $B$ является диамагнитным, а ион $\mathrm{Co}^{2+}$ в узле $A$ находится в высокоспиновом состоянии $(S=3 / 2)$.

Работа выполнена при поддержке Российского Фонда фундаментальных исследований (грант № 17-02-01068).

DOI: 10.21883/FTT.2019.03.47245.300

\section{1. Введение}

Поиск новых магнитных нецентральных примесей, которые могут одновременно приводить к возникновению сегнетоэлектрического и магнитного порядка и магнитоэлектрическому взаимодействию в кристаллах [1], является в настоящее время актуальной задачей. Материалы, обладающие этими свойствами, относятся к мультиферроикам - многофункциональным материалам, открывающим новые возможности для современной электроники и спинтроники.

Очевидно, что для получения мультиферроидных свойств необходимо, чтобы материал обладал магнитным моментом, например, за счет его легирования магнитными примесями. Положение атома в кристаллической решетке и его локальное окружение могут существенно влиять на величину магнитного момента иона. Для атомов $3 d$ переходных элементов, кроме того, характерна возможность существования их в нескольких зарядовых состояниях, которые зависят от положения атома в решетке, его локального окружения (изолированный атом, комплексы с различными дефектами) и присутствия в образцах других доноров и акцепторов.

Влиять на зарядовое и спиновое состояние можно изменяя условия синтеза. Так, в случае примеси Мn в $\mathrm{SrTiO}_{3}$ было показано, что на зарядовое состояние и соотношение числа атомов в разных узлах решетки можно влиять, изменяя температуру отжига и стехиометрию образцов (см. [2,3] и ссылки в этих работах). Наши исследования легированного никелем титаната стронция показали, что магнитное состояние Ni зависит от того, какие примесные комплексы он образует при легировании. В случае комплекса $\mathrm{Ni}^{2+}-V_{\mathrm{O}}$ спиновое состояние никеля зависит от расстояния между примесью и вакансией (ближайшая или удаленная вакансия) [4,5].
Недавние эксперименты показали, что $\mathrm{SrTiO}_{3}$, легированный кобальтом с концентрацией $14-40 \%$, является ферромагнетиком при $300 \mathrm{~K}$ и одновременно диэлектриком $[6,7]$, что встречается сравнительно редко. Это было одной их причин постановки настоящих исследований. Предварительное изучение этого материала указывало на возможность вхождения примеси Со в разные узлы структуры перовскита [8].

Титанат стронция, легированный кобальтом, исследуется давно. Близость кристаллических структур $\mathrm{SrTiO}_{3}$ и $\mathrm{SrCoO}_{3-x}$ (перовскит с дефектностью по кислороду) позволяет предположить существование в этой системе непрерывного ряда твердых растворов. Согласно [9], растворимость кобальта в узле $B$ в $\mathrm{SrTiO}_{3}$ составляет по крайней мере $40 \%$, а в работе [10] сообщалось о приготовлении методом твердофазного синтеза поликристаллических образцов, содержащих до 90\% Со. Рентгеновские исследования в последней работе обнаружили при $x>0.5$ появление дополнительных пиков на рентгенограммах, которые связывались с упорядочением вакансий кислорода. Во всех опубликованных ранее работах предполагалось, что примесный атом кобальта замещает атом титана. Никаких сведений о вхождении кобальта в узел $A$ не было.

Для определения зарядового состояния кобальта в $\mathrm{SrTiO}_{3}(\mathrm{Co})$ использовались методы ЭПР, рентгеновской фотоэлектронной спектроскопии (XPS), оптическое поглощение, а также изучение околокраевой структуры в спектрах рентгеновского поглощения (XANES). По данным ЭПР [11] на монокристаллах с $0.2 \%$ Со наблюдался сигнал от ионов $\mathrm{Co}^{4+}$ в низкоспиновом состоянии $(S=1 / 2)$, причем величина сигнала резко увеличивалась при предварительном освещении. По-видимому, в 
исходном состоянии примесь кобальта в образце является диамагнитной и находится в зарядовом состоянии $\mathrm{Co}^{3+}$. Действительно, исследования спектров оптического поглощения на монокристаллах в работах $[11,12]$ обнаружили линии поглощения, характерные для иона $\mathrm{Co}^{3+}$ в октаэдрическом окружении. Исследования методом XPS тонких пленок с концентрацией кобальта от 10 до $50 \%$, полученных методом молекулярно-лучевой эпитаксии (МЛЭ) при $550^{\circ} \mathrm{C}$ [7], позволили сделать вывод о зарядовом состоянии кобальта $2+$. Вывод о том же самом зарядовом состоянии на основании исследований XPS был сделан и для нановолокон $\mathrm{SrTiO}_{3}(\mathrm{Co})$ c концентрацией кобальта до $20 \%$, полученных методом электроспиннинга и отожженных при $650^{\circ} \mathrm{C}$ [13], а также для образцов с $30 \%$ Со, полученных методом импульсного лазерного напыления (PLD) [6]. Анализ данных XPS позволил авторам [6] предположить, что примесные атомы Со замещают ионы Ті с образованием вакансий кислорода и что зарядовое состояние Со в пленках ниже, чем в объемных образцах, поскольку пленки выращивались при низком парциальном давлении кислорода. (Поскольку зарядовое состояние кобальта в узле $B$ отличается от заряда иона титана, это предполагает существование в образце компенсирующих дефектов, в частности, вакансий кислорода.)

Следует заметить, что поскольку для атома Со разброс энергий фотоэлектронных пиков превышает величину химического сдвига при изменении зарядового состояния [14], то вывод о его зарядовом состоянии +2 в $\mathrm{SrTiO}_{3}$ в [6,7] был сделан по появлению сильного высокоэнергичного сателлитного пика. Однако этот же пик с несколько меньшей интенсивностью присутствует в образцах с трехвалентным кобальтом [15], и поэтому надежность этого способа определения зарядового состояния вызывает вопросы. Косвенным аргументом в пользу появления $\mathrm{Co}^{2+}$ в пленках, полученных методом PLD, может служить рост параметра решетки, перпендикулярного плоскости пленки, с увеличением концентрации кобальта [6]. Этот эффект явно отличается от уменьшения параметра решетки при легировании, наблюдаемого в объемных образцах $[10,16]$. К сожалению, из спектров XANES, записанных на пленках $\mathrm{SrTiO}_{3}(\mathrm{Co})$ [6], никаких выводов о зарядовом состоянии кобальта сделано не было.

Для объяснения причин появления ферромагнетизма при комнатной температуре был проведен ряд теоретических исследований $[7,17,18]$, в которых возможная природа этого явления обсуждалась на основе вывода о присутствии в образцах двухвалентного кобальта. Эти исследования показали существенную роль вакансий кислорода, образующих комплексы ион $\mathrm{Co}^{2+}-$ ближайшая вакансия, который обладает спиновым моментом.

Исследования магнитных свойств легированных кобальтом образцов $\mathrm{SrTiO}_{3}$ показало, что методы объемного синтеза обычно не приводят к появлению ферромагнетизма при комнатной температуре $[10,16]$. В работе [16] в однофазных образцах $\mathrm{SrTi}_{1-x} \mathrm{Co}_{x} \mathrm{O}_{3}$ при $x=0.35-0.50$ однако наблюдался другой переход из парамагнитного в антиферромагнитное состояние при 15-26K. Рентгеновские измерения этих образцов, восстановленных в атмосфере $10 \% \mathrm{H}_{2}-\mathrm{Ar}$, обнаружили в них присутствие металлического Со. Напротив, тонкие пленки легированного кобальтом $\mathrm{SrTiO}_{3}$ с диэлектрическими или полупроводниковыми свойствами, выращенные методами PLD или МЛЭ, показывают ферромагнетизм при $300 \mathrm{~K}$ при высокой концентрации примеси $(>14 \%)[6,7,19]$. Легированные кобальтом тонкие пленки $(\mathrm{La}, \mathrm{Sr}) \mathrm{TiO}_{3}$ с металлической проводимостью, отожженные в восстановительной атмосфере, демонстрируют ферромагнетизм при $300 \mathrm{~K}$ при существенно более слабом легировании кобальтом $(\sim 2 \%)[20,21]$.

Обращает на себя внимание то, что свойства образцов существенно зависят от метода их получения. Для образцов, полученных при высоких температурах, характерно наличие трехвалентного кобальта и отсутствие ферромагнетизма. Напротив, в условиях, которые в известной степени можно рассматривать как далекие от равновесия (гидротермальный синтез, лазерное напыление, нановолокна), появляются двухвалентный кобальт и ферромагнетизм при $300 \mathrm{~K}$. Это делает весьма актуальной задачу определения реальной структуры образуемых кобальтом примесных дефектов.

В настоящей работе методами XAFS-спектроскопии исследовано структурное положение и зарядовое состояние магнитной примеси $\mathrm{Co}$ в образцах $\mathrm{SrTiO}_{3}$, полученных методом твердофазного синтеза с различным отклонением от стехиометрии. Показано, что в зависимости от условий получения атомы кобальта могут входить преимущественно либо в узлы $B$ структуры перовскита в трехвалентном состоянии, либо в узлы $A$ в двухвалентном состоянии, образуя в последнем случае нецентральную примесь в высокоспиновом состоянии. Полученные результаты сопоставляются с результатами расчетов структуры дефектов, проведенных из первых принципов.

\section{2. Образцы, методика эксперимента и расчетов}

Образцы $\mathrm{SrTiO}_{3}$, легированные кобальтом с концентрацией примеси 2-3\% и различным отклонением от стехиометрии, получались методом твердофазного синтеза. Исходными компонентами служили $\mathrm{SrCO}_{3}$, нанокристаллический $\mathrm{TiO}_{2}$, полученный гидролизом тетрапропилортотитаната и высушенный при $500^{\circ} \mathrm{C}$, и $\mathrm{Co}\left(\mathrm{NO}_{3}\right)_{2} \cdot 6 \mathrm{H}_{2} \mathrm{O}$. Компоненты взвешивались в необходимых пропорциях, перетирались в ацетоне и отжигались на воздухе в корундовых тиглях при $1100^{\circ} \mathrm{C}$ в течение $4 \mathrm{~h}$. Полученные порошки снова перетирались и повторно отжигались в тех же условиях в течение $4 \mathrm{~h}$. Часть образцов дополнительно отжигалась на воздухе при 1500 или $1600^{\circ} \mathrm{C}$ в течение $2 \mathrm{~h}$. Для введения кобальта в узлы $A$ или $B$ структуры перовскита состав 
образцов преднамеренно отклонялся от стехиометрии в сторону избытка титана или стронция. Все полученные образцы были темно-коричневого цвета.

Рентгеновские спектры поглощения в областях протяженной тонкой структуры (EXAFS) и околокраевой структуры (XANES) записывались в режиме флуоресценции в окрестности $K$-края поглощения Сo $(7.709 \mathrm{keV})$ при $300 \mathrm{~K}$ на станции КМС-2 на источнике синхротронного излучения BESSY (Германия). Излучение монохроматизировалось двухкристальным монохроматором $\mathrm{Si}_{1-x} \mathrm{Ge}_{x}$ с ориентацией (111). Интенсивность падающего на образец излучения измерялась с помощью ионизационной камеры, интенсивность флуоресцентного излучения - кремниевым дрейфовым детектором RÖNTEC, работавшим в энергодисперсионном режиме.

Спектры EXAFS обрабатывались с помощью широко используемого программного пакета IFEFFIT [22]. Функция EXAFS $\chi(k)$ (здесь $k=\sqrt{2 m\left(E-E_{0}\right)} / \hbar-$ волновой вектор фотоэлектрона, $E_{0}$ - энергия края поглощения) выделялась из экспериментальных спектров с помощью программы ATHENA и подгонялась с помощью программы ARTEMIS к теоретической кривой, рассчитанной для заданной структурной модели

$$
\begin{aligned}
\chi(k)= & -\frac{1}{k} \sum_{j} \frac{N_{j} S_{0}^{2}}{R_{j}^{2}}\left|f_{j}(k)\right| \\
& \times \exp \left[-2 \sigma_{j}^{2} k^{2}-2 R_{j} / \lambda(k)\right] \sin \left[2 k R_{j}+\phi_{j}(k)\right],
\end{aligned}
$$

где суммирование проводится по нескольким ближайшим координационным сферам $j$ вокруг центрального атома $\mathrm{Co}, N_{j}$ - число атомов в $j$-й координационной сфере, $R_{j}$ - ее радиус, $f_{j}-$ амплитуда рассеяния, $\lambda-$ эффективная длина свободного пробега, $\sigma_{j}^{2}-$ фактор Дебая-Уоллера, характеризующий среднеквадратичную амплитуду отклонения расстояния до атомов сорта $j$ от средней величины, а $S_{0}^{2}-$ множитель, учитывающий уменьшение амплитуды осцилляций за счет многочастичных эффектов. Амплитуда рассеяния $f_{j}(k)$, фазовые сдвиги $\phi_{j}(k)$ и длина свободного пробега $\lambda(k)$ для всех путей однократного и многократного рассеяния рассчитывались с помощью программы FEFF6. Для каждого образца записывалось 3-4 спектра, которые затем независимо обрабатывались, а полученные зависимости $\chi(k)$ усреднялись. Детали обработки данных приведены в [23].

Расчеты из первых принципов геометрии, магнитной и электронной структуры примесных центров проводились с помощью программы ABINIT в приближении $\mathrm{LDA}+U$ на 80-атомных кубических сверхъячейках, в которых один из ионов $\mathrm{Ti}^{4+}$ в узле $B$ или $\mathrm{Sr}^{2+}$ в узле $A$ структуры перовскита замещался ионом кобальта. Для описания атомов с частично заполненной $d$-оболочкой использовались PAW-псевдопотенциалы. Значения параметров, описывающих кулоновское и обменное взаимодействие внутри $d$-оболочки, составляли $U=5$ и $J=0.9 \mathrm{eV}$.

\section{3. Экспериментальные результаты}

Рентгенофазовый анализ полученных образцов показал, что все исследованные образцы $\mathrm{SrTiO}_{3}(\mathrm{Co})$ были однофазными и имели кубическую структуру перовскита при $300 \mathrm{~K}$. Типичные дифрактограммы трех образцов показаны на рис. 1, а найденные для них параметры решетки и (для сравнения) параметр решетки нелегированного $\mathrm{SrTiO}_{3}$ - в табл. 1. Уменьшение параметра решетки всех легированных образцов по сравнению с нелегированным $\mathrm{SrTiO}_{3}$ согласуется с ранее опубликованными данными $[10,16]$ и указывает на образование твердого раствора.

Для определения зарядового состояния примеси Со в легированных образцах $\mathrm{SrTiO}_{3}$ положение края поглощения в спектрах XANES исследованных образцов сравнивалось с положением краев в эталонных образцах $\mathrm{LaCoO}_{3}$ (валентность кобальта +3 ) и $\mathrm{Co}\left(\mathrm{NO}_{3}\right)_{2} \cdot 6 \mathrm{H}_{2} \mathrm{O}$ (валентность кобальта +2). Спектры XANES всех образцов и эталонных соединений показаны на рис. 2.

Сравнение спектров XANES всех образцов $\mathrm{SrTiO}_{3}(\mathrm{Co})$, отожжённых при температуре $1100^{\circ} \mathrm{C}$, a также образца, отожжённого при $1500^{\circ} \mathrm{C}$ с отклонением от стехиометрии в сторону избытка стронция, показало, что края поглощения в этих образцах близки между собой и практически совпадают с краем поглощения эталонного образца $\mathrm{LaCoO}_{3}$. Это позволяет сделать вывод о том, что примесь Со в этих образцах находится преимущественно в зарядовом состоянии +3 .

Края поглощения образцов $\mathrm{SrTiO}_{3}(\mathrm{Co})$ с отклонением от стехиометрии в сторону избытка Ti, отожжённых при температурах 1500 и $1600^{\circ} \mathrm{C}$, совпадают между собой и близки к краю поглощения в эталонном образце $\mathrm{Co}\left(\mathrm{NO}_{3}\right)_{2} \cdot 6 \mathrm{H}_{2} \mathrm{O}$. Это указывает на то, что в этих образцах кобальт находится преимущественно в зарядовом состоянии +2 .

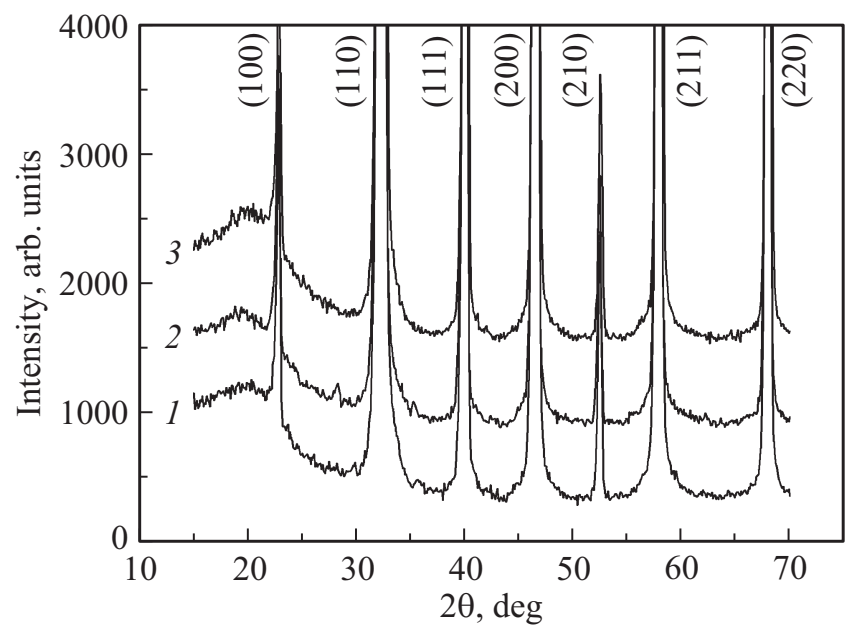

Рис. 1. Дифрактограммы образцов: 1 - образец $\mathrm{Sr}_{0.98} \mathrm{Co}_{0.02} \mathrm{TiO}_{3}$, отожженный при $1600^{\circ} \mathrm{C}, 2$ - образец $\mathrm{Sr}_{0.98} \mathrm{Co}_{0.02} \mathrm{TiO}_{3}$, отожженный при $1500^{\circ} \mathrm{C}, 3$ - образец $\mathrm{SrTi}_{0.97} \mathrm{Co}_{0.03} \mathrm{O}_{3}$, отожженный при $1500^{\circ} \mathrm{C}$. 
Таблица 1. Параметры решетки трех образцов $\mathrm{SrTiO}_{3}(\mathrm{Co})$ и нелегированного $\mathrm{SrTiO}_{3}$

\begin{tabular}{c|c|c|c|c}
\hline Образцы & $\begin{array}{c}\mathrm{Sr}_{0.98} \mathrm{Co}_{0.02} \mathrm{TiO}_{3}, \\
\text { отжиг } 1600{ }^{\circ} \mathrm{C}\end{array}$ & $\begin{array}{c}\mathrm{Sr}_{0.98} \mathrm{Co}_{0.02} \mathrm{TiO}_{3}, \\
\text { отжиг } 1500^{\circ} \mathrm{C}\end{array}$ & $\begin{array}{c}\mathrm{SrTi}_{0.97} \mathrm{Co}_{0.03} \mathrm{O}_{3}, \\
\text { отжиг } 1500^{\circ} \mathrm{C}\end{array}$ & $\mathrm{SrTiO}_{3}$ \\
\hline Параметр решетки $a, \AA$ & $3.8977 \pm 0.0005$ & $3.8994 \pm 0.0002$ & $3.8931 \pm 0.0006$ & 3.905
\end{tabular}

Следует заметить, что химический сдвиг в спектрах XANES намного больше, чем в фотоэлектронных спектрах [14], что позволяет делать выводы о зарядовом состоянии примеси Со более обоснованно.

Для определения структурного положения примеси $\mathrm{Co}$ в $\mathrm{SrTiO}_{3}$ был проведен анализ спектров EXAFS. При анализе данных и выборе структурной модели учитывались данные рентгеновских и XANES-исследований, а именно параметр решетки и возможность атомов Со входить в разные узлы ( $A$ и $B)$ структуры перовскита, в том числе, одновременно и в разных зарядовых состояниях.

Сравнение спектров EXAFS для двух групп образцов, в которых ионы Со имеют разное зарядовое состояние, показало их качественное различие.

Для всех образцов, в которых Со находится в зарядовом состоянии $\mathrm{Co}^{3+}$, неплохое согласие между экспериментальными спектрами и теоретически рассчитанными кривыми $\chi(k)$ получалось для модели, в которой ион $\mathrm{Co}^{3+}$ замещал ион $\mathrm{Ti}^{4+}$ с образованием удаленной вакансии кислорода. В этой модели расстояние Со-О в первой координационной сфере составляло $1.909 \pm 0.012 \AA$, расстояние $\mathrm{Co}-\mathrm{Sr}-3.342 \pm 0.016 \AA$, а расстояние $\mathrm{Co}-\mathrm{Ti}-3.892 \pm 0.012 \AA$.

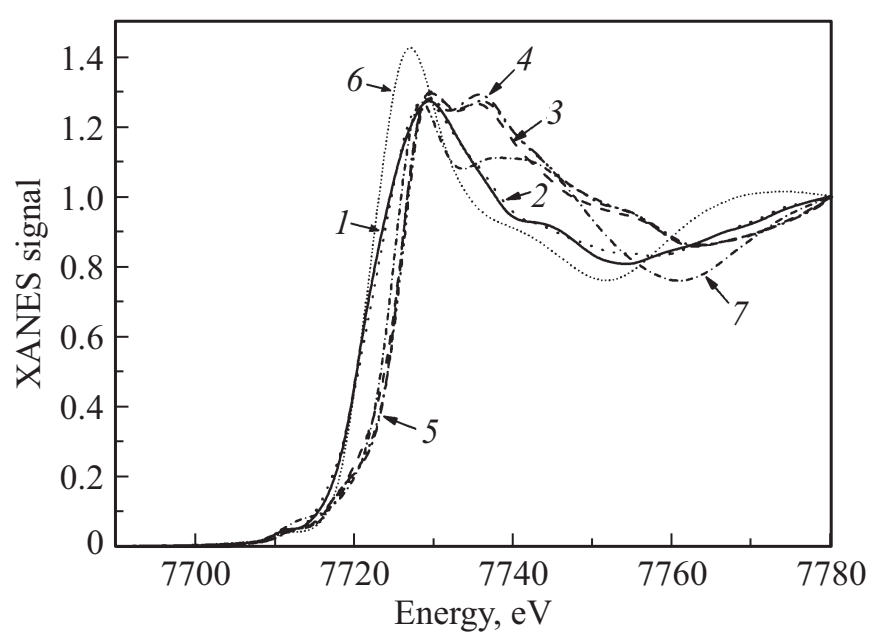

Рис. 2. Спектры XANES для образцов $\mathrm{SrTiO}_{3}(\mathrm{Co})$ и эталонных соединений кобальта: 1 - образец $\mathrm{Sr}_{0.98} \mathrm{Co}_{0.02} \mathrm{TiO}_{3}$, отожженный при $1600^{\circ} \mathrm{C}, 2$ - образец $\mathrm{Sr}_{0.98} \mathrm{Co}_{0.02} \mathrm{TiO}_{3}$, отожженный при $1500^{\circ} \mathrm{C}, 3$ - образец $\mathrm{Sr}_{0.98} \mathrm{Co}_{0.02} \mathrm{TiO}_{3}$, отожженный при $1100^{\circ} \mathrm{C}, 4$ - образец $\mathrm{SrTi}_{0.97} \mathrm{Co}_{0.03} \mathrm{O}_{3}$, отожженный при $1500^{\circ} \mathrm{C}, 5-$ образец $\mathrm{SrTi}_{0.97} \mathrm{Co}_{0.03} \mathrm{O}_{3}$, отожженный при $1100^{\circ} \mathrm{C}, 6-\mathrm{Co}\left(\mathrm{NO}_{3}\right)_{2} \cdot 6 \mathrm{H}_{2} \mathrm{O}, 7-\mathrm{LaCoO}_{3}$.
Анализ спектров EXAFS для образцов $\mathrm{Sr}_{0.98} \mathrm{Co}_{0.02} \mathrm{TiO}_{3}$, отожженных при 1600 и $1500^{\circ} \mathrm{C}$, в которых примесный атом Со находится в зарядовом состоянии +2 , показал, что ни одна из моделей, в которых ион кобальта $\mathrm{Co}^{2+}$ замещает либо $\mathrm{Ti}^{4+}$ в узле $B$, либо $\mathrm{Sr}^{2+}$ в узле $A$, не дает удовлетворительного согласия с экспериментом. Качественное согласие между экспериментальным спектром и теоретически рассчитанной кривой EXAFS для этих образцов получалось для модели, в которой ион $\mathrm{Co}^{2+}$ замещал ион $\mathrm{Sr}^{2+}$ в узле $A$, смещаясь из узла в направлении [100] на $\sim 1.0 \AA \AA$, что представляется разумным с учетом большой разницы ионных радиусов ионов $\mathrm{Co}^{2+}$ и $\mathrm{Sr}^{2+}$. Расстояние от атома кобальта до ближайших атомов кислорода при этом составляет $1.993 \pm 0.042 \AA$.

К сожалению, согласие между экспериментальными и теоретически рассчитанными кривыми EXAFS для всех исследуемых образцов не было достаточно хорошим (критерием согласия расчетных и экспериментальных спектров считались малое значение $R$-фактора, соответствие найденных координационных чисел теоретической модели и согласие фурье-трансформант спектров в $R$-представлении). Поэтому было решено рассмотреть микроскопические модели различных примесных центров с атомом Со в узлах $A$ и $B$.

\section{4. Результаты расчетов из первых принципов}

При построении теоретических моделей учитывались данные о зарядовом состоянии примесных атомов и межатомные расстояния, полученные из анализа спектров EXAFS. Заметим, что проведенные ранее расчеты из первых принципов $[7,17,18]$ были выполнены в основном для двухвалентного кобальта в узле $B$, в то время как полученные нами результаты указывают на другие зарядовые состояния и другие узлы решетки.

Моделирование геометрии примесных центров было начато с иона $\mathrm{Co}^{2+}$ в узле $A$. Этот примесный центр является одним из наиболее простых, поскольку замещение стронция кобальтом не требует никакой электрической компенсации. Расчеты с учетом полной релаксации окружающих атомов показали, что узловое положение иона $\mathrm{Co}^{2+}$ в узле $A$ в $\mathrm{SrTiO}_{3}$ энергетически неустойчиво и примесный атом переходит в нецентральное положение. Сравнение энергий структур с атомами, смещенными вдоль направлений [100], [110] и [111], 
показало, что наименьшую энергию имеет структура со смещением вдоль оси [110], а энергии структур со смещениями [100] и [111] соответственно на 264 и $985 \mathrm{meV}$ выше. Величина смещения составляет 1.03, 1.00 и $0.79 \AA$ А соответственно для смещений вдоль [100], [110] и [111], а спин иона во всех конфигурациях равен $S=3 / 2$. Расчетные расстояния до ближайших атомов в окружении кобальта для указанных конфигураций представлены в табл. 2. Видно, что расчетные расстояния $\mathrm{Co}-\mathrm{O}$ до ближайших атомов кислорода заметно превышают расстояния Со-О для Со в узле $B$, а наиболее близкой к полученной из эксперимента величине $1.993 \pm 0.042 \AA$ оказываются межатомные расстояния для смещений вдоль направлений [100] и [111].

Моделирование примесных центров с трехвалентным кобальтом в узле $B$ предполагает существование дефектов, компенсирующих разность зарядов ионов. Расчеты для изолированного иона $\mathrm{Co}^{3+}$ в узле $B$, в котором дополнительный электрон поставлялся удаленным донорным атомом F в узле кислорода (табл. 2), показали, что наименышую энергию имеет низкоспиновое состояние $(S=0)$. Сравнение полученных расстояний для модели,
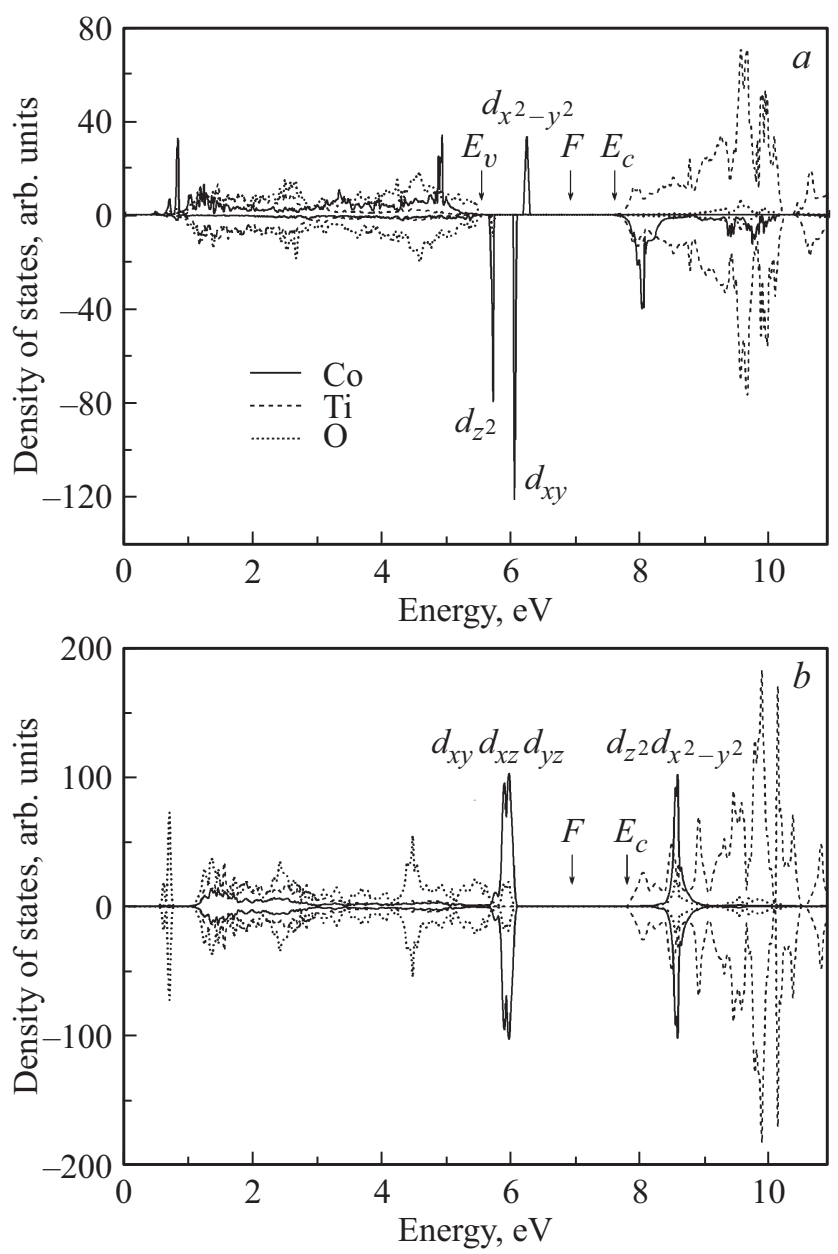

Pис. 3. Парциальные вклады кобальта, титана и кислорода в плотность состояний для примесных центров $\mathrm{Co}_{A}^{2+}(a)$ и $\mathrm{Co}_{B}^{3+}(b)$. Парциальный вклад кобальта уменьшен в 5 раз.
Таблица 2. Локальное окружение ионов Со в различных теоретических моделях

\begin{tabular}{|c|c|c|c|c|c|c|c|}
\hline \multirow{2}{*}{ Модель } & \multirow{2}{*}{$S$} & \multicolumn{6}{|c|}{ Координационная сфера } \\
\hline & & 1 & 2 & 3 & 4 & 5 & 6 \\
\hline $\begin{array}{l}\text { Нецентральный } \\
\text { атом в узле } A \text {, } \\
\text { смещение }[100]\end{array}$ & $3 / 2$ & $\begin{array}{c}2.014 \\
(4 \mathrm{O})\end{array}$ & $\begin{array}{l}2.948 \\
(4 \mathrm{Ti})\end{array}$ & $\begin{array}{l}3.056 \\
(1 \mathrm{Sr})\end{array}$ & $\begin{array}{l}3.168 \\
(4 \mathrm{O})\end{array}$ & $\begin{array}{l}3.630 \\
(4 \mathrm{O})\end{array}$ & $\begin{array}{l}3.814 \\
(4 \mathrm{O})\end{array}$ \\
\hline $\begin{array}{l}\text { Нецентральный } \\
\text { атом в узле } A \text {, } \\
\text { смещение }[110]\end{array}$ & $3 / 2$ & $\begin{array}{l}2.051 \\
(1 \mathrm{O})\end{array}$ & $\begin{array}{l}2.069 \\
(4 \mathrm{O})\end{array}$ & $\begin{array}{l}2.706 \\
(2 \mathrm{Ti})\end{array}$ & $\begin{array}{l}3.265 \\
(2 \mathrm{O})\end{array}$ & $\begin{array}{l}3.318 \\
(2 \mathrm{Sr})\end{array}$ & $\begin{array}{l}3.474 \\
(4 \mathrm{Ti})\end{array}$ \\
\hline $\begin{array}{l}\text { Нецентральный } \\
\text { атом в узле } A \text {, } \\
\text { смещение }[111]\end{array}$ & $3 / 2$ & $\begin{array}{c}1.996 \\
(3 \mathrm{O})\end{array}$ & $\begin{array}{l}2.707 \\
(1 \mathrm{Ti})\end{array}$ & $\begin{array}{l}2.946 \\
(6 \mathrm{O})\end{array}$ & $\begin{array}{l}3.216 \\
\text { (3Ti) }\end{array}$ & $\begin{array}{l}3.523 \\
(3 \mathrm{Sr})\end{array}$ & $\begin{array}{l}3.564 \\
(3 \mathrm{O})\end{array}$ \\
\hline $\begin{array}{l}\text { Изолированный } \\
\text { ион } \mathrm{Co}^{3+} \text { в узле } B\end{array}$ & 0 & $\begin{array}{l}1.905 \\
(6 \mathrm{O})\end{array}$ & $\begin{array}{l}3.352 \\
(8 \mathrm{Sr})\end{array}$ & $\begin{array}{l}3.801 \\
(6 \mathrm{Ti})\end{array}$ & $\begin{array}{l}4.355 \\
(24 \mathrm{O})\end{array}$ & $\begin{array}{l}5.509 \\
(12 \mathrm{Ti})\end{array}$ & \\
\hline
\end{tabular}

в которой ион $\mathrm{Co}^{3+}$ входит в узел $B$, с полученным в эксперименте расстоянием $1.909 \pm 0.012 \AA$ показывает, что они неплохо согласуются между собой.

Электронная структура примесного центра - иона $\mathrm{Co}^{2+}$ в узле $A-$ показана на рис. $3, a$. Видно, что примесь кобальта создает три акцепторных уровня в запрещенной зоне $\mathrm{SrTiO}_{3}$, связанных с занятыми электронами орбиталями $d_{x^{2}-y^{2}}$ (спин вверх) и $d_{z^{2}}$ и $d_{x y}$ (спин вниз). Уровень Ферми $F$ при этом лежит в запрещенной зоне. Состояние $d_{z^{2}}$ сильно гибридизовано с $p$-состояниями кислорода, поэтому разрешенные правилами отбора оптические переходы с этого уровня на дно зоны проводимости $E_{c}$, формируемой $d$-состояниями кобальта и титана, могут быть причиной интенсивной окраски образцов.

Для центра - изолированного иона $\mathrm{Co}^{3+}$ в узле $B-$ $d$-уровни кобальта симметрии $T_{2 g}$ сливаются с краем валентной зоны, а уровни симметрии $E_{g}$ образуют резонансные уровни в зоне проводимости (рис. 3,b). Поэтому заметного изменения окраски образцов в этом случае ожидать не приходится.

\section{5. Уточнение структурных моделей и обсуждение результатов}

Недостаточно хорошее согласие экспериментальных и расчетных спектров EXAFS, достигнутое в разделе 3, побудило нас рассмотреть более сложные модели. Так, была рассмотрена модель, в которой кобальт в узле $B$ образует комплекс с ближайшей вакансией кислорода. $\mathrm{B}$ рамках этой модели для образца $\mathrm{SrTi}_{0.97} \mathrm{Co}_{0.03} \mathrm{O}_{3}$, отожжённого при температуре $1100^{\circ} \mathrm{C}$, были получены расстояния, мало отличающиеся от приведенных выше, однако параметр $S_{0}^{2}$ оказался ближе к значению этого параметра в эталонных соединениях. 
Таблица 3. Структурные параметры, полученные из обработки спектров EXAFS двух исследованных образцов

\begin{tabular}{|c|c|c|c|c|c|c|}
\hline Образец & Модель & $R$-фактор & $S_{0}^{2}$ & Коорд. сфера & $R_{i}, \AA$, эксп. & $\sigma_{i}^{2}, \AA^{2}$ \\
\hline \multirow{2}{*}{$\begin{array}{c}\mathrm{Sr}_{0.98} \mathrm{Co}_{0.02} \mathrm{TiO}_{3}, \\
\text { отжиг } 1600^{\circ} \mathrm{C}\end{array}$} & Узел $A$ & \multirow{2}{*}{0.00297} & 0.705 & $\begin{array}{l}R_{\mathrm{Co}-\mathrm{O}} \\
R_{\mathrm{Co}-\mathrm{Ti}} \\
R_{\mathrm{Co}-\mathrm{Sr}}\end{array}$ & $\begin{array}{c}2.040(6) \\
3.094(15) \\
2.816(20)\end{array}$ & $\begin{array}{l}0.0049(18) \\
0.0146(27) \\
0.0114(40)\end{array}$ \\
\hline & Узел $B$ & & 0.282 & $\begin{array}{l}R_{\mathrm{Co}-\mathrm{O}} \\
R_{\mathrm{Co}-\mathrm{Sr}} \\
R_{\mathrm{Co}-\mathrm{Ti}}\end{array}$ & $\begin{array}{l}1.906(11) \\
3.354(19) \\
3.907(17)\end{array}$ & $\begin{array}{l}0.0037(18) \\
0.0087(12) \\
0.0064(13)\end{array}$ \\
\hline \multirow{2}{*}{$\begin{array}{c}\mathrm{SrTi}_{0.97} \mathrm{Co}_{0.03} \mathrm{O}_{3}, \\
\text { отжиг } 1100^{\circ} \mathrm{C}\end{array}$} & Узел $A$ & \multirow{2}{*}{0.00255} & 0.153 & $\begin{array}{l}R_{\mathrm{Co}-\mathrm{O}} \\
R_{\mathrm{Co}-\mathrm{Ti}} \\
R_{\mathrm{Co}-\mathrm{Sr}}\end{array}$ & $\begin{array}{c}2.040(6) \\
3.094(15) \\
2.816(20)\end{array}$ & $\begin{array}{l}0.0049(18) \\
0.0146(27) \\
0.0114(40)\end{array}$ \\
\hline & Узел $B$ & & 0.731 & $\begin{array}{l}R_{\mathrm{Co}-\mathrm{O}} \\
R_{\mathrm{Co}-\mathrm{Sr}} \\
R_{\mathrm{Co}-\mathrm{Ti}}\end{array}$ & $\begin{array}{l}1.906(11) \\
3.354(19) \\
3.907(17)\end{array}$ & $\begin{array}{l}0.0037(18) \\
0.0087(12) \\
0.0064(13)\end{array}$ \\
\hline
\end{tabular}

Используя результаты расчета геометрии локального окружения нецентрального атома кобальта в узле $A$ для трех направлений смещения, был обработан спектр образца $\mathrm{Sr}_{0.98} \mathrm{Co}_{0.02} \mathrm{TiO}_{3}$, отожженного при $1600^{\circ} \mathrm{C}$. Оказалось, что наименьшие значения $R$-фактора при обработке кривых дают конфигурации с направлением смещения [100] и [111]. В расчетной фурье-трансформанте спектра EXAFS для модели со смещением вдоль [110] появляется сильный пик от атомов Ті в третьей координационной сфере, который полностью отсутствует в экспериментальном спектре. Поэтому направление смещения [110] можно полностью исключить из дальнейшего рассмотрения. Учитывая значительную разницу энергий конфигураций со смещением [100] и [111], мы склонны считать, что смещение атомов происходит в направлении [100], и будем в дальнейшем рассматривать эту модель.

Далее мы предположили, что в реальных образцах атомы кобальта могут одновременно замещать атомы в узлах $A$ и $B$. Определение структурных параметров для двух составляющих спектра EXAFS, которые мы будем называть состояниями $A$ и $B$ и в которых атомы примеси находятся в соответствующих узлах решетки, проводилось следующим способом. В качестве начального приближения предполагалось, что спектр образца $\mathrm{SrTi}_{0.97} \mathrm{Co}_{0.03} \mathrm{O}_{3}$, отожженного при $1100^{\circ} \mathrm{C}$, представляет собой спектр „чистого“ состояния $B$. Моделирование локального окружения кобальта в узле $B$ проводилось с учетом ближайшей вакансии кислорода. Для этой модели находился набор структурных параметров (расстояния и факторы Дебая-Уоллера для трех ближайших координационных сфер). Спектр образца $\mathrm{Sr}_{0.98} \mathrm{Co}_{0.02} \mathrm{TiO}_{3}$, отожженного при $1600^{\circ} \mathrm{C}$, представлялся в виде суммы состояний $A$ и $B$ с неизвестными относительными долями. Зафиксировав определенные на предыдущем шаге параметры состояния $B$ и взяв в качестве начального приближения межатомные расстояния, рассчитанные из первых принципов для состояния $A$ (см. разд. 4), на- ходились структурные параметры для состояния $A$ и относительные доли состояний $A$ и $B$. Далее, считая, что в образце $\mathrm{SrTi}_{0.97} \mathrm{Co}_{0.03} \mathrm{O}_{3}$, отожженном при $1100^{\circ} \mathrm{C}$, может присутствовать небольшая доля состояния $A$, проводилась повторная обработка спектра этого образца. Теперь уже фиксировались данные, определенные на предыдущем этапе для состояния $A$. Это давало уточненные параметры для состояния $B$ и относительные доли состояний $A$ и $B$ в этом образце. Многократное повторение описанной итерационной процедуры позволило найти структурные параметры состояний $A$ и $B$ и их относительные доли в анализируемых спектрах.

Типичный экспериментальный спектр EXAFS и его наилучшая аппроксимация в рамках описанного подхода показаны на рис. 4, а межатомные расстояния до ближайших координационных сфер для образцов, отожженных при 1100 и $1600^{\circ} \mathrm{C}$, приведены в табл. 3. Полученные из эксперимента межатомные расстояния

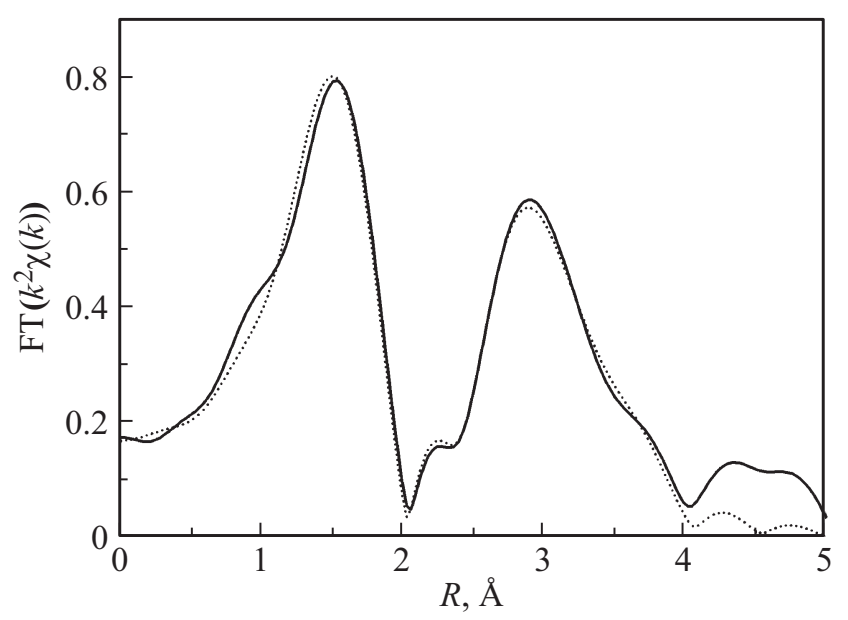

Рис. 4. Сравнение амплитуд фурье-трансформант для типичного спектра EXAFS образца $\mathrm{Sr}_{0.98} \mathrm{Co}_{0.02} \mathrm{TiO}_{3}$ (отжиг $1600^{\circ} \mathrm{C}$, сплошная линия) и расчетной кривой (пунктирная линия), отвечающей наилучшему согласию. 
согласуются с расчетными (ср. табл. 2 и 3). По данным итерационной процедуры при температуре отжига $1600^{\circ} \mathrm{C}$ в образце $\mathrm{Sr}_{0.98} \mathrm{Co}_{0.02} \mathrm{TiO}_{3}$ в узле $A$ оказывается 76\% введенной примеси кобальта, а в образце $\mathrm{SrTi}_{0.97} \mathrm{Co}_{0.03} \mathrm{O}_{3}$ при температуре отжига $1100^{\circ} \mathrm{C}$ в узле $A$ находится около $18 \%$ примесных атомов.

\section{6. Заключение}

В настоящей работе представлены результаты исследований локальной структуры и зарядового состояния примеси кобальта в $\mathrm{SrTiO}_{3}$ методом XAFS-спектроскопии. Обнаружено, что при определенных условиях синтеза кобальт может входить в узел $A$ структуры перовскита. Меняя условия синтеза, можно заметно изменять соотношение концентраций атомов кобальта, входящих в узлы $A$ и $B$. Так, при температуре отжига $1600^{\circ} \mathrm{C}$ в узел $A$ входит $76 \%$ атомов Со, а при температуре $1100^{\circ} \mathrm{C}$ - только $18 \%$.

Исследование спектров XANES показало, что зарядовое состояние кобальта в узле $A$ равно +2 , а в узле $B-$ +3 . Установлено, что сдвиг края поглощения в спектрах XANES намного более чувствителен к изменению зарядового состояния кобальта по сравнению с методом XPS. Исследования EXAFS установили, что атомы Со в узле $A$ являются нецентральными, а их смещение составляет $\sim 1.0 \AA$. Согласно проведенным расчетам из первых принципов, значение магнитного момента иона $\mathrm{Co}_{A}^{2+}$ равно $S=3 / 2$. Таким образом, ионы $\mathrm{Co}_{A}^{2+}$ в $\mathrm{SrTiO}_{3}$ являются еще одним представителем группы магнитных нецентральных примесей, с которыми могут быть связаны интересные мультиферроидные свойства.

\section{Список литературы}

[1] V.V. Shvartsman, S. Bedanta, P. Borisov, W. Kleemann, A. Tkach, P.M. Vilarinho. Phys. Rev. Lett. 101, 165704 (2008).

[2] А.И. Лебедев, И.А. Случинская, А. Ерко, В.Ф. Козловский. Письма в ЖЭТФ 89, 545 (2009).

[3] И.А. Случинская, А.И. Лебедев, А. Ерко. Изв. РАН. Сер. физ., 74, 1289 (2010).

[4] I.A. Sluchinskaya, A.I. Lebedev, A. Erko. J. Adv. Dielectrics 3, 4, 1350031 (2013).

[5] И.А. Случинская, А.И. Лебедев, А. Ерко. ФТТ, 56, 442 (2014).

[6] L. Bi, H.-S. Kim, G.F. Dionne1, C.A. Ross. New J. Phys. 12, 043044 (2010).

[7] A.B. Posadas, C. Mitra, C. Lin, A. Dhamdhere, D.J. Smith, M. Tsoi, A.A. Demkov. Phys. Rev. B 87, 144422 (2013).

[8] A.I. Lebedev, I.A. Sluchinskaya. In: Fundamental Physics of Ferroelectrics and Related Materials 2016 Workshop. Abstracts. Carnegie Institution for Science, Washington (2016) P. 211-212.

[9] A. Murashkina, V. Maragou, D. Medvedev, V. Sergeeva, A. Demin, P. Tsiakaras. J. Power Sources 210, 339 (2012).

[10] S. Malo, A. Maignan. Inorg. Chem. 43, 8169 (2004).

[11] K.W. Blazey, K.A. Muller. J. Phys. C 16, 5491 (1983).
[12] Я. Дойчилович, Н. Кулагин, Д. Попович, С. Спасович. Кристаллография 49, 534 (2004).

[13] W. Zhang, H.-P. Li, W. Pan. J. Mater. Sci. 47, 8216 (2012).

[14] NIST X-ray Photoelectron Spectroscopy Database; https://srdata.nist.gov/

[15] A. Chainani, M. Mathew, D.D. Sarma. Phys. Rev. B 46, 9976 (1992).

[16] C. Pascanut, N. Dragoe, P. Berthet. J. Magn. Magn. Mater. 305, 6 (2006).

[17] J.M. Florez, S.P. Ong, M.C. Onbaşli, G.F. Dionne, P. Vargas, G. Ceder, C.A. Ross. Appl. Phys. Lett. 100, 252904 (2012).

[18] C. Mitra, C. Lin, A.B. Posadas, A.A. Demkov. Phys. Rev. B 90, 125130 (2014).

[19] S.X. Zhang, S.B. Ogale, D.C. Kundaliya, L.F. Fu, N.D. Browning, S. Dhar, W. Ramadan, J.S. Higgins, R.L. Greene, T. Venkatesan. Appl. Phys. Lett. 89, 012501 (2006).

[20] Y.G. Zhao, S.R. Shinde, S.B. Ogale, J. Higgins, R.J. Choudhary, V.N. Kulkarni, R.L. Greene, T. Venkatesan. Appl. Phys. Lett. 83, 2199 (2003).

[21] G. Herranz, R. Ranchal, M. Bibes, H. Jaffrès, E. Jacquet, J.L. Maurice, K. Bouzehouane, F. Wyczisk, E. Tafra, M. Basletic, A. Hamzic, C. Colliex, J.P. Contour, A. Barthélémy, A. Fert. Phys. Rev. Lett. 96, 027207 (2006).

[22] IFEFFIT home page; http://cars9.uchicago.edu/ifeffit/

[23] A.I. Lebedev, I.A. Sluchinskaya, V.N. Demin, I.H. Munro. Phys. Rev. B 55, 14770 (1997).

Редактор Т.Н. Василевская 